This report was prepared as an account of work sponsored by an agency of the United States Government. Neither the United States Government nor any agency thereof, nor any of their employees, makes any warranty, express or implied, or assumes any legal liability or responsibility for the accuracy, completeness, or usefulness of any information, apparatus, product, or process disclosed, or represents that its use would not infringe privately owned rights. Reference herein to any specific commercial product, process, or service by trade name, trademark, manufacturer, or otherwise does not necessarily constitute or imply its endorsement, recommendation, or favoring by the United States Government or any agency thereof. The views and opinions of authors expressed herein do not necessarily state or reflect those of the United States Government or any agency thereof.

\author{
SAND95.0742C
}

\title{
HIGH-SPEED OPTICAL STUDIES OF THE DRIVING PLASMA IN LASER ACCELERATION OF FLYER PLATES*
}

\author{
Wayne M. Trott \\ Sandia National Laboratories, Albuquerque, NM 87185-0834
}

\begin{abstract}
High-speed optical methods have been used to study several characteristics of the high-temperature driving plasma in laser acceleration of flyers with optical fiber coupling. Flyer materials examined include pure aluminum and composite targets containing an insulating layer of $\mathrm{Al}_{2} \mathrm{O}_{3}$. Results from fast-gating spectroscopy show that silicon appears early in the plasma spectrum, indicating that the coupling fiber material plays an active role in flyer acceleration. Plasma emission persists longer and pressure broadening of discrete lines decays more slowly in the launch of composite flyers. Fast-gating spectroscopy has also been used in conjunction with very thin ("trace") metal layers deposited in the target fabrication process to examine the depth of ablation by the driving plasma.
\end{abstract}

\section{INTRODUCTION}

Laser-driven acceleration of flyer plates affords a promising approach to well-controlled, short-pulse shock compression of condensed phase materials. With this application in mind, the generation and characterization of laser-accelerated flyers have been subjects of active investigation (1-8). The use of step-index, multimode optical fibers for coupling laser energy to the flyer target offers several advantages in optimization of this experimental technique (1) and has been exploited in detailed studies of flyer velocity-time histories as a function of incident fluence, optical pulse duration, target area and thickness, etc. (3). These experiments in combination with parallel theoretical studies $(4,5)$ have shown that a modest laser driver can deliver predictable and precisely variable flyer impactor velocities over a wide range of experimental parameters. For many practical operating conditions, simple scaling laws adequately describe the dependence of final flyer velocity on several of these parameters, including incident fluence, target mass, etc.

The dynamic physical behavior of laser-driven flyers is an additional, crucial factor in determining

*This work performed at Sandia National Laboratories supported by the U. S. Department of Energy under contract DE-AC0494AL85000. their utility in shock compression studies. Tests with pulsed-laser stereo photography (6) and other high-speed imaging techniques $(6,7)$ have shown that excellent overall flyer planarity can generally be obtained; however, the acceleration process can give rise to a number of complex, multidimensional effects that are evident on a small scale. For example, very early in the launch process, the flyer surface typically exhibits a fine-structure nonplanarity that is consistent with the apparent spatial frequency of modal noise in the optical intensity distribution at the fiber output (7). Reflecting changes in spatial properties of the evolving plasma, these small-scale perturbations grow significantly with time. Rapid heat transfer from the high-temperature plasma to the accelerating flyer can also lead to melt and vaporization of a significant fraction of the plate material $(9,10)$. The vital role of the expanding plasma in laser acceleration of flyers motivates direct studies of this aspect of the launch process.

In this paper, we describe recent observations of the driving plasma, including broadband measurements of the time-dependent intensity of plasma emission in combination with spectral data obtained with a fast-gating optical multichannel analyzer (OMA) system. Characteristics of the plasma generated in the launch of pure aluminum flyers are compared to those observed with composite materi- 
als containing an insulating layer of $\mathrm{Al}_{2} \mathrm{O}_{3}$. This comparison is of interest in view of the well-established $(2,8)$ superior performance of composite flyers/impactors (i.e., consistently higher peak velocity vs. incident optical fluence and larger amplitude and duration of pressure pulses delivered to an acceptor). Fast-gating spectroscopy has also been used to examine the plasma generated in acceleration of targets containing very thin layers of different ("trace") metals deposited with the usual materials. These tests suggest a useful approach for examination of the depth of flyer ablation by the plasma.

\section{EXPERIMENTAL}

Important elements of the experimental design used in this work are shown schematically in Fig. 1. The setup for flyer generation using optical fiber coupling has been described in detail previously $(1,3)$. The driving laser was a Q-switched Nd:Glass oscillator (Lasermetrics Model 9380). The laser output $(\lambda=1.054 \mu \mathrm{m})$ was horizontally polarized and multimode. Laser energy was focused by a $100-\mathrm{mm}$ focal length lens and coupled into the large-diameter $(1000 \mu \mathrm{m})$ end of a fiber taper that was positioned so that the diverging beam slightly underfilled the input face. This taper concentrated the optical energy into a $400-\mu \mathrm{m}$-diameter fiber section. From this element, the energy was proximity coupled either to a thin $(0.5 \mathrm{~mm})$ fused silica substrate coated (at the output face) with the flyer target material or to a $1-\mathrm{m}$-long fiber prepared in a similar manner. The different target samples (described below) were fabricated by physical vapor deposition. Absorbing neutral density filters were used to vary the energy incident on the flyer targets. All tests were performed with laser pulse durations near $18 \mathrm{~ns}$ (FWHM).

As shown in Fig. 1, the optical diagnostics were configured to examine plasma emission collected and transmitted in the "backward" direction (i.e.,opposite to that of the driving laser propagation path) by the fiber elements. In addition to providing some degree of spatial resolution (defined by the numerical aperture of the final fiber), this sampling scheme favored the detection of fiber material (e.g., $\mathrm{Si}, \mathrm{O}$ ) in the plasma. Direct evidence of such species was sought in order to test computational results which indicate that the fiber compound plays a substantial

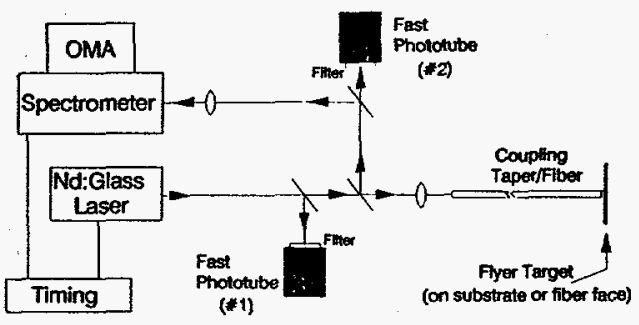

FIGURE 1. Schematic diagram of experimental design for optical studies of the driving plasma in laser acceleration of flyers.

role in the ablation process driving the flyer target (10). UV-transmitting optics were used to facilitate detection of strong $\mathrm{Si}$ emission features in the 2500-3000 A region. Plasma light was directed to the entrance slit of a $0.33-\mathrm{m}$ spectrometer. The dispersed spectrum was viewed by a silicon target vidicon coupled to a gateable proximity focused channel intensifier tube and the signal was processed by the OMA. The detector was gated on by a voltage pulse, which was triggered at variable delay with respect to the driving laser pulse by using digital delay generators. Gates as short as $10 \mathrm{~ns}$ could be obtained; however, data collection over 60-ns intervals was sufficient to follow the most important changes in the plasma spectrum. The temporal profile of plasma intensity was measured by a fast photodetector (time constant $<0.4 \mathrm{~ns}$ ). For this diagnostic, appropriate broadband filters were used to isolate the desired region of the spectrum and to discriminate against reflected laser light.

\section{RESULTS AND DISCUSSION}

Figure 2 presents phototube records of visible plasma light generated in the launch of two different flyer targets: (a) an 8- $\mu \mathrm{m}$-thick film of pure Al and (b) an 8- $\mu \mathrm{m}$-thick composite film with an embedded $0.25-\mu \mathrm{m}$-thick layer of $\mathrm{Al}_{2} \mathrm{O}_{3}$ placed $0.25 \mu \mathrm{m}$ from the film/fiber interface. In both cases, the emission intensity was observed to rise abruptly near the time corresponding to the onset of flyer motion. Comparable peak intensities were obtained with the two materials but emission persisted for a significantly longer time in acceleration of the composite film. Higher peak intensities and shorter 


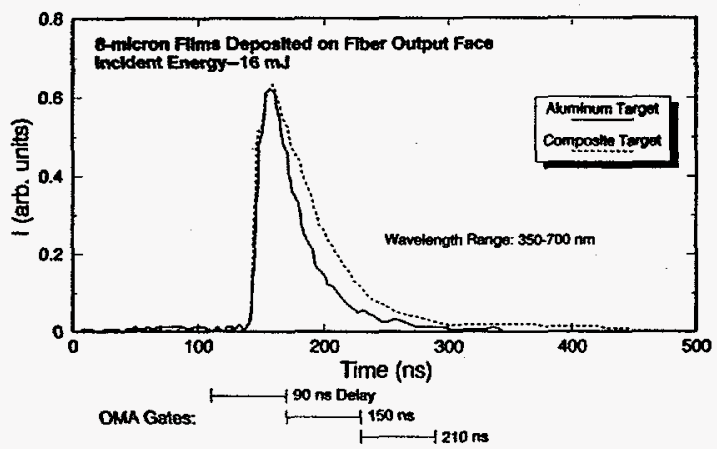

FIGURE 2. Temporal profile of plasma emission intensity observed in the acceleration of two different flyer materials. OMA gates (60-ns intervals) used in spectral studies are shown in relationship to the emission records.

emission "pulses" were seen in tests on very thin (2$\mu \mathrm{m}) \mathrm{Al}$ and composite films. Again, a longer pulse was observed in the latter case. Relatively long-lived emission appears to be a characteristic feature of composite flyer acceleration since similar results were obtained in othër wavelength bands as well as with different flyer thicknesses. Definitive interpretation of this phenomenon is difficult due to the limited spatial resolution and wavelength bandpass of the data, uncertain optical thickness of the plasma, etc. It is likely that the thermal confinement of the insulating $\mathrm{Al}_{2} \mathrm{O}_{3}$ layer results in the plasma remaining hotter for a slightly longer time. Also, interactions giving rise to pressure broadening occur over a longer time in the composite flyer case (as demonstrated below).

Changes in the spectral properties (2550-3150 A region) of plasma driving aluminum and composite films are illustrated in Fig. 3 and Fig. 4, respectively. The relationship of the nominal delay times cited in these figures to the time-dependent emission intensity are shown in Fig. 2. Hence, the gate designated "90 ns delay" captured light in the first $\sim 30$ ns of flyer acceleration, etc. For both flyer types, the early spectrum is dominated by continuum emission, a reasonable result in view of the high particle densities and field strengths in the plasma during this time. Discrete emission features from vaporized and ionized material appear at later intervals, as the plasma rapidly expands. Identified features include an $\mathrm{Al}$ multiplet $(3082 \AA, 3093 \AA$ ), a Si line at $2882 \AA$, and a Si ${ }^{+}$line at $2906 \mathrm{~A}(11,12)$. Similar features have been seen in other wavelength regions; e.g., a Si mul-

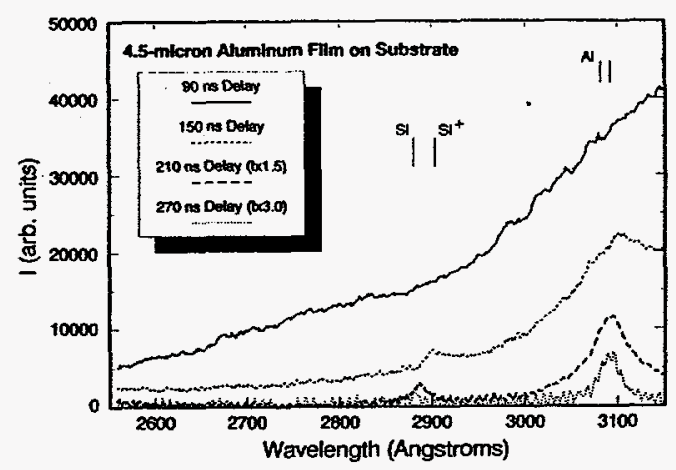

FIGURE 3. Spectra of emission from plasma driving an Al film deposited on a fused silica substrate; $15 \mathrm{~mJ}$ incident energy. Each curve represent $s$ the sum of six individual shots.

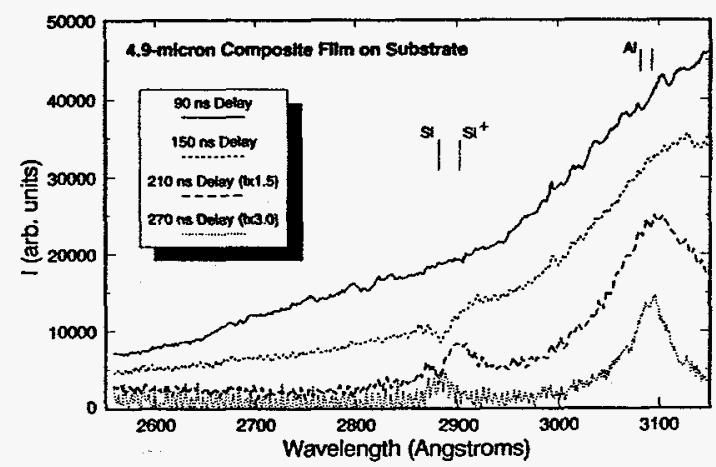

FIGURE 4. Spectra of emission from plasma driving a composite film deposited on a fused silica substrate; $15 \mathrm{~mJ}$ incident energy. Each curve represents the sum of six individual shots.

tiplet near $2520 \AA, \mathrm{a} \mathrm{Si}^{+}$line at $4131 \AA$, and strong Al lines at $3944 \AA$ and $3962 \AA$, respectively.

In accordance with the phototube data, the overall intensity of plasma emission falls off more quickly in the launch of a pure Al flyer. Pressure broadening of the $\mathrm{Al}$ lines clearly decays faster in this event, as well. This result suggests that material associated with the plasma remains more dense and compact during acceleration of a composite target, an interpretation consistent with other data on the visual appearance of the region behind the flyer in both cases, as observed by back lit shadowgraphy (7) and an image motion camera technique (8).

Of particular interest is the definite identification of silicon features, indicating substantial vaporization and ionization of the fiber material. During the earliest stages of acceleration, the high particle density, etc. in the plasma seems to preclude direct 


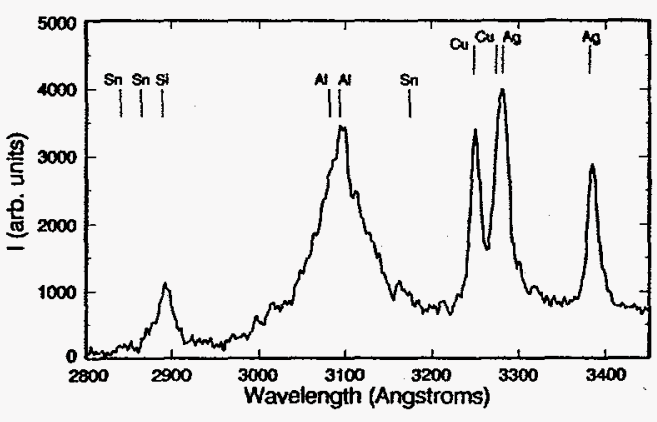

FIGURE 5. Late-time spectrum of emission from plasma driving an Al flyer target containing embedded $0.01-\mu \mathrm{m}$-thick layers of copper, silver and tin; $15 \mathrm{~mJ}$ incident energy.

resolution of these features. Their relatively early appearance, however, lends strong support to the concept that the fiber core can be a major participant in pushing the flyer, as predicted by recent computational work (10). Initially, $\mathrm{Si}^{+}$is seen in emission while $\mathrm{Si}$ is observed in absorption (cf. Figs. 3 and 4). Si absorption also occurs in the $2520 \AA$ region during this time. Later, $\mathrm{Si}$ alone is observed in emission. This time-dependent behavior is entirely consistent with the expected temperature fields near the fiber/ target interface. As the fiber compound begins to participate in the driving plasma, temperature increases from the "cold" fiber to a region of vaporized material, with still higher temperatures occurring in the ionized regime. At late times, expansion cools the plasma so that only neutrals remain in emission.

The utility of thin layers of different metals as a diagnostic approach for the study of flyer ablation and thinning is demonstrated in Fig. 5. The prepared target (4- $\mu \mathrm{m}$ total thickness) consisted of aluminum embedded with $0.01-\mu \mathrm{m}$ layers of copper, silver, and tin. These layers were positioned at $0.10 \mu \mathrm{m}, 0.21$ $\mu \mathrm{m}$, and $0.32 \mu \mathrm{m}$ from the fiber/target interface, respectively. Prominent lines of all relevant materials appear in the 2800-3400 $\AA$ region, facilitating acquisition of a useful spectrum on a single shot. Important features include two $\mathrm{Cu}$ lines (3248 $\AA, 3274 \AA$ ), two $\mathrm{Ag}$ lines $(3281 \AA, 3383 \AA)$ and three Sn lines $(2840 \AA, 2863 \AA, 3175 \AA)$. The $\mathrm{Cu}$ and $\mathrm{Ag}$ features are very strong, implying vaporization to a depth of at least $0.22 \mu \mathrm{m}$. The relatively weak $\mathrm{Sn}$ lines may reflect less vaporization at $0.33 \mu \mathrm{m}$; however, lower intensities for these features are also consistent with published line strengths (11). Spectra acquired from samples with embedded layers of copper and silver at depths of $0.25 \mu \mathrm{m}$ and $0.5 \mu \mathrm{m}$, respectively, exhibit strong $\mathrm{Cu}$ lines and very weak $\mathrm{Ag}$ features. This result suggests that ablation can involve $>0.5 \mu \mathrm{m}$ of the flyer target. The substantial vaporization depths observed here are once again in good agreement with computational results (10).

\section{ACKNOWLEDGMENTS}

The excellent technical assistance of Jaime $\mathrm{N}$. Castañeda is gratefully acknowledged. Flyer targets were skillfully prepared by Catharine Sifford (Thin Film \& Brazing Team, SNL).

\section{REFERENCES}

1. Trott, W. M., and Meeks, K. D., "Acceleration of Thin Foil Targets Using Fiber-Coupled Optical Pulses," in Shock Woves in Condensed Matter--1989, eds. S. C. Schmidt, et al., New York: Elsevier Science Publishers, 1990, pp. 997-1000.

2. D. L. Paisley, "Laser-Driven Miniature Flyer Plates for Shock Initiation of Secondary Explosives," in Shock Waves in Condensed Matter-1989, eds. S. C. Schmidt, et al., New York: Elsevier Science Publishers, 1990, pp. 733-736.

3. Trott, W. M., "Studies of Laser-Driven Flyer Acceleration Using Optical Fiber Coupling," in Shock Waves in Condensed Matter--1991, eds. S. C. Schmidh, et al., New York: Elsevier Science Publishers, 1992, pp. 829-832.

4. Farnsworth, A. V., and Lawrence, R. J., "Numerical and Analytical Analysis of Thin Laser-Driven Flyer Plates," in Shock Waves in Condensed Matter-1991, eds. S. C. Schmidt, et al., New York: Elsevier Science Publishers, 1992, pp. 821-824.

5. Lawrence, R. J., and Trott, W. M., "Theoretical Analysis of a Pulsed-Laser-Driven Hypervelocity Flyer Launcher," Int. J. Impact Engineering 14, 439-449 (1993).

6. Paisley, D. L., Montoya, N. I., Stahl, D. B., and Garcia, I. A., "Interferometry, Streak Photography, and Stereo Photography of Laser-Driven Miniature Flyer Plates," in SPIE Proceedings No. 1358, Cambridge, UK, 1990, pp. 760-765.

7. Frank, A. M., and Trott, W. M., "Stop Motion Microphotography of Laser Driven Plates," in SPIE Proceedings No. 2273, San Diego, CA, 1994, pp. 196-206.

8. Trott, W. M., "Investigation of the Dynamic Behavior of Laser-Driven Flyers," in High-Pressure Science and Technology-1993, eds. S. C. Schmidt, et al., New York: AIP Press, 1994, pp. 1655-1658.

9. Frank, A. M., and Trott, W. M., "Investigation of Thin LaserDriven Flyer Plates Using Streak Imaging and Stop-Motion Microphotography," (this volume).

10. Farnsworth, Jr., A. V., "Laser Acceleration of Thin Flyers" (this volume).

11. Meggers, W. F., Corliss, C. H., and Scribner, B. F., Tables of Spectral-Line Intensities, 2nd Edition, Washington: National Bureau of Standards, 1975.

12. Lanz, T., and Artru, M.-C., Physica Scripta 32, 115-124 (1985). 